\title{
Corrigendum
}

\section{Corrigendum to "Role of the immune system in pancreatic cancer progression and immune modulating treatment strategies" [Cancer Treat. Rev. 40 (2014) 513-522]}

\author{
K. Sideras ${ }^{\text {a }}$, H. Braat ${ }^{\text {}}$, J. Kwekkeboom ${ }^{\text {c }}$, C.H. van Eijck ${ }^{d}$, M.P. Peppelenbosch ${ }^{\text {e }}$, S. Sleijfer ${ }^{\text {f }}$, M. Bruno ${ }^{\text {g,* }}$ \\ a Erasmus University Medical Center, Department of Gastroenterology and Hepatology, NA-0621's, Gravendijkwal 230, 3015 CE Rotterdam, The Netherlands \\ ${ }^{\mathrm{b}}$ Erasmus University Medical Center, Department of Gastroenterology and Hepatology, Hs-510's, Gravendijkwal 230, 3015 CE Rotterdam, The Netherlands \\ ' Erasmus University Medical Center, Laboratory of Gastroenterology and Hepatology, NA-1009's, Gravendijkwal 230, 3015 CE Rotterdam, The Netherlands \\ ${ }^{\mathrm{d}}$ Erasmus University Medical Center, Department of Surgery, Room H-818k's, Gravendijkwal 230, 3015 CE Rotterdam, The Netherlands \\ e Erasmus University Medical Center, Laboratory of Gastroenterology and Hepatology, Na-1007's, Gravendijkwal 230,3015 CE Rotterdam, The Netherlands \\ ${ }^{\mathrm{f}}$ Erasmus University Medical Center, Department of Oncology, He-116's, Gravendijkwal 230, 3015 CE Rotterdam, The Netherlands \\ ${ }^{\mathrm{g}}$ Erasmus University Medical Center, Department of Gastroenterology and Hepatology, H-358's, Gravendijkwal 230, 3015 CE Rotterdam, The Netherlands
}

The authors regret that the sentence beginning on line 12 of first column on page 516 , relating to Reference 43 is misleading.

The sentence reads:

"At the same time however, Gal-1 is found overexpressed on the stromal tissues of long term pancreatic cancer survivors [43], indicating that the prognostic significance of Gal-1 expression is not entirely clear".
However, the authors would like to correct this as:

"At the same time, Gal-1 is found underexpressed on the stromal tissues of long term pancreatic cancer survivors [43], indicating that the prognostic significance of Gal-1 expression is promising".

The authors would like to apologise for any inconvenience caused.

\footnotetext{
DOI of original article: http://dx.doi.org/10.1016/j.ctrv.2013.11.005

* Corresponding author. Tel.: +31 107035946; fax: +31 107030352.

E-mail address: m.bruno@erasmusmc.nl (M. Bruno).
} 